\title{
CIRCULARLY CONFIGURED MULTI-SLM HOLOGRAPHIC DISPLAY SYSTEM
}

\author{
Fahri Yaraş, Hoonjong Kang, Levent Onural
}

\author{
Department of Electrical and Electronics Engineering, Bilkent University, TR-06800 Ankara, Turkey \\ fahri@ee.bilkent.edu.tr, hjkang@ee.bilkent.edu.tr, onural@ee.bilkent.edu.tr
}

\begin{abstract}
The designed circular holographic display system produces ghostlike 3D optical reconstructions of a computer generated 3D model. System uses six phase-only reflective-type spatial light modulators (SLMs) that are configured circularly. Alignment of the SLMs are successful and gap problem is solved by using half-mirrors. The total number of pixels of the resultant display is $11520 \times 1080$. Reconstructions show that increase in the viewing angle is significant compared to the single SLM case. With the help of the proposed system, observer can see the reconstructions binocularly. As a result, comfortable 3D perception is achieved. In order to avoid eyehazard, LED illumination is also used as an alternative light source. Experimental results are satisfactory. Proposed system can be used as a holographic display system.
\end{abstract}

Index Terms - holographic displays, spatial light modulators, phase holograms, three-dimensional displays, computer generated holography, holographic 3DTV

\section{INTRODUCTION}

Holographic displays are one of the candidate systems for future 3DTV applications. Since holography can reproduce volume filling light fields, it is a convenient technique to optically duplicate 3D objects. Those ghost-like images can be seen without any optical aid. There is no need for special glasses or eye-tracking systems. In addition, since holography regenerates optical duplicates of the 3D objects, observers do not feel motion sickness or headaches as in most of the stereoscopic displays.

Electro-holographic displays are reported in the literature. [1, 2, $3,4,5,6,7,8,9,10]$. There are several pixelated devices which are suitable for digital holographic display systems. Liquid crystal (LC) displays, liquid crystal on silicon (LCoS) displays, digital micromirror devices are a few of them. Since all those pixelated devices modulate the incoming light in the spatial domain, either transmissively or reflectively, they are called spatial light modulators (SLMs). Full complex modulators would be the ultimate solution; however, most of the spatial light modulators usually modulate only the amplitude or the phase of the incident light. In our holographic display prototypes we use phase-only LCoS SLMs.

Although those SLMs are easy to use, have high diffraction efficiency and are rich in term of pixel count, they are quite small in size. Typical phase-only SLM size is approximately $1 \mathrm{~cm} \times 2 \mathrm{~cm}$. As a result of it, reconstructions are also quite small. It is almost impossible to see the reconstructions binocularly and rotate around them when only one such SLM is used. Therefore researchers are trying to come up with ideas to overcome this bottleneck. Maeno et al. proposed a method to increase the size of the holographic display

978-1-61284-162-5/11/\$26.00 (C) 2011 IEEE

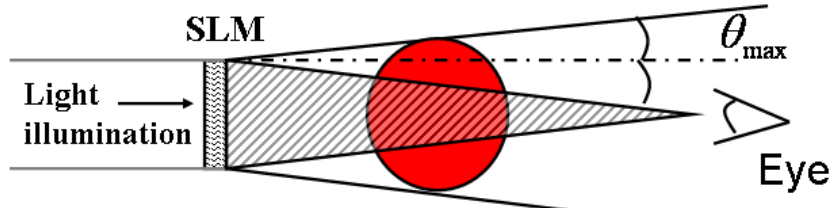

\section{Viewable area}

Fig. 1. Illustration of larger object reconstruction with a single SLM.

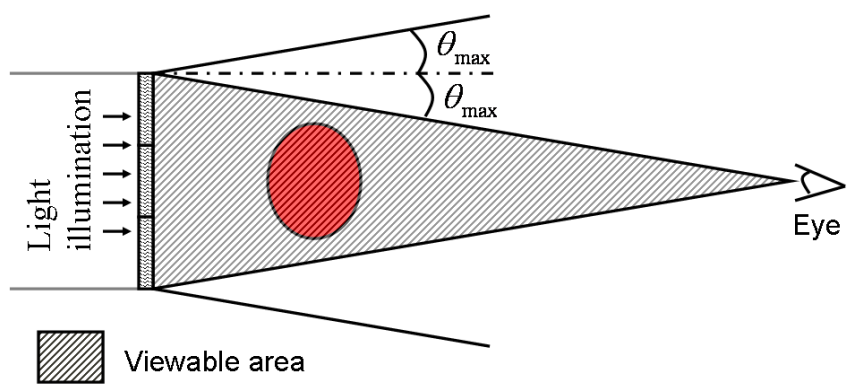

Fig. 2. Illustration of larger object reconstruction with multiple SLMs.

system and also the field of view [11]. In their system they use five transmissive type SLMs and place them side by side. They increase the field of view along the horizontal direction, but they discard the vertical parallax by using a lenticular sheet. Yet another holographic display system is reported by Hahn et al. and they use mirror modules and other optical components to increase the size of the display system [12]. Their horizontal parallax only (HPO) curved holographic display system provides approximately $23^{\circ}$ viewing angle. A company from Germany, called SeeReal Technologies, developed a novel technique $[13,14,15,16,17]$. With the help of the technique, only a part of the wavefront that enters to the eye pupil of the observer is reconstructed.

Different from the above systems, we propose to build a fullparallax circularly configured multi-SLM holographic display system. We use six phase-only reflective type spatial light modulators to increase the horizontal size of the holographic display. With the help of the proposed system, observers can see ghost-like 3D images floating in the space binocularly with naked eye. Methods and configurations for enlargement of the viewing angle will be presented in the following section. Section 3 presents the optical setup and hologram generation algorithm. Experimental results will be presented in Section 4. Conclusions will be drawn in the last section. 


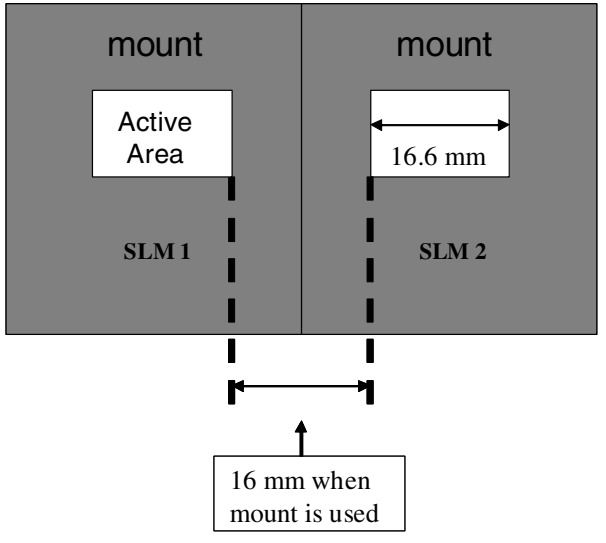

Fig. 3. Gap between two SLMs when they are aligned side by side

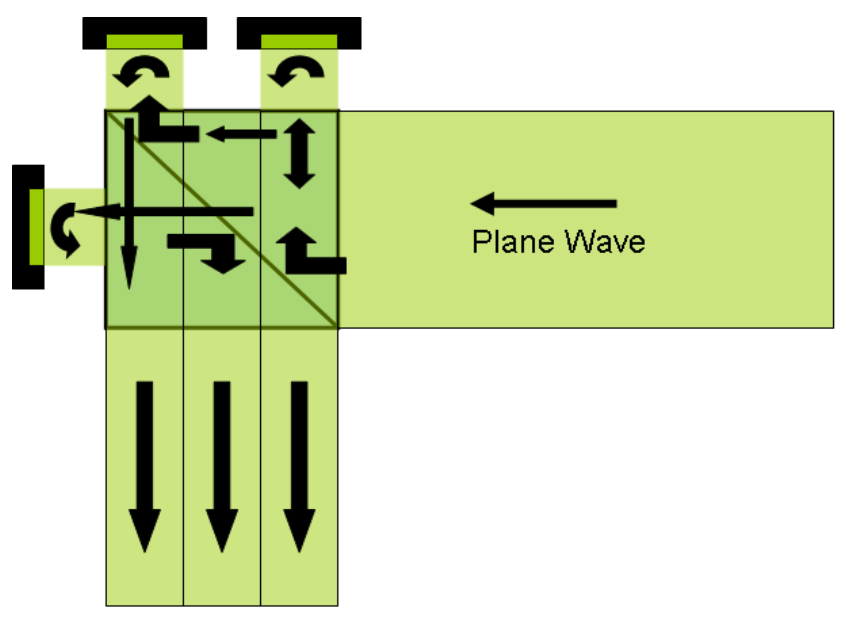

Fig. 4. Alignment of three SLMs to have a continuous array without any gap

\section{ENLARGEMENT OF THE VIEWING ANGLE}

For the pixelated devices, local maximum diffraction angle for Shannon (low-pass filter) recovery case can be calculated as:

$$
\theta_{\max }=\sin ^{-1}\left(\lambda f_{\max }\right)
$$

where;

$$
f_{\max }=\frac{1}{2 \Delta_{p}}
$$

Here $\Delta_{p}$ denotes the pixel period of the device, $\lambda$ is the wavelength of the incident light. Within the limit of the local maximum frequency, we can reconstruct 3D objects larger than the SLM size [3]. Those reconstructions can be seen on a diffuser or a screen. However, in order to see the full reconstruction by naked eye, 3D object should be in the viewable area (See Fig. 1). Otherwise observer can only see a part of it. One way to solve this problem is to increase the hologram size (See Fig. 2). Since viewable area is increased, observer can see larger reconstructions.

However, since SLMs have hard mount around it, when we place them side by side a large gap occurs between two SLMs (See Fig. 3). There are methods to overcome this problem, such as using mirror modules as in [12]. In our system, we propose to use multiple beam

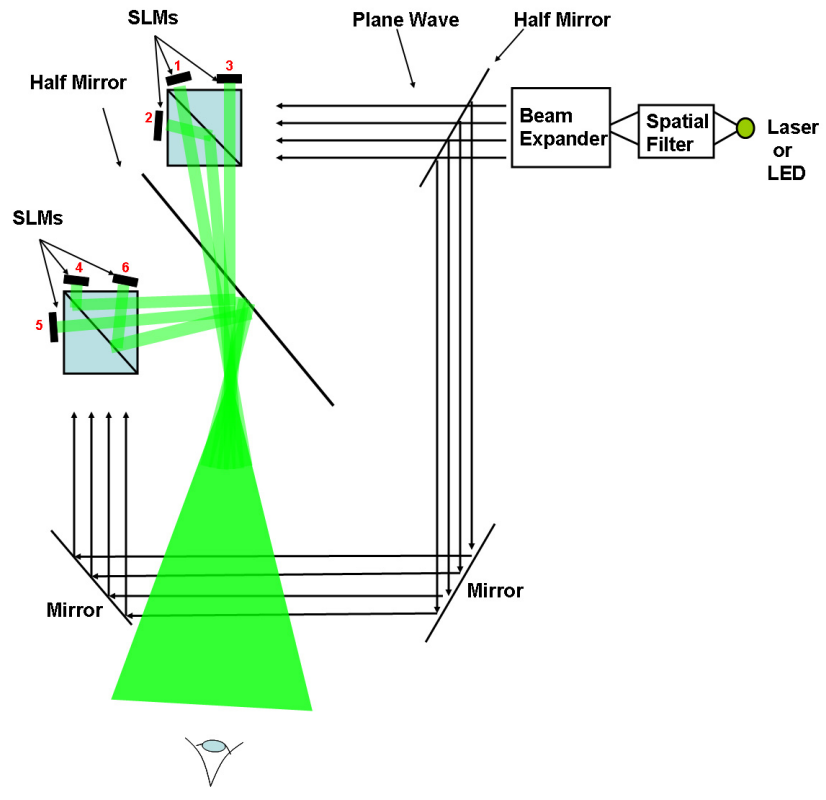

Fig. 5. Schematic of the setup

splitters (half-mirrors). As seen in Fig. 4, with the help of the beamsplitters, the gap between two SLMs are filled by the image of the third SLM. The same configuration is applied to other three SLMs and finally, these two sets are combined with a large beam-splitter (See Fig. 5). Therefore the resultant hologram size increases six times along the horizontal direction. This gives a freedom to the observer to see the reconstructions binocularly and to rotate within the limit of the viewing angle. Fig. 5 also shows the increase in the viewing angle. This enlargement is illustrated by the shaded area at the observer side. Observer can move around the holographic reconstruction in this shaded area without experiencing any discontinuity in the reconstruction.

\section{OPTICAL SETUP AND HOLOGRAM GENERATION}

Schematic of the setup can be seen in Fig. 5. The method to obtain a larger hologram size along the horizontal direction is described in the previous section. After aligning the six SLMs side by side, we rotate the SLMs with the help of high precision stages to configure them circularly. Each SLM is rotated by $1^{\circ}$ with respect to the next SLM (this can be increased up to $3^{\circ}$ ). Fine adjustments are also needed after the rotations. Adjustments are crucial. First, centers of all SLMs should be on the same curve and there should not be any gap between the SLMs. Second, holographic reconstructions should be aligned. In order to be sure that the alignments of the reconstructions are satisfactory we load a hologram of a single point to all SLMs. The reconstruction distance of the point has to be chosen to be the same with the desired distance of the holographic reconstruction (the reconstruction distance is $400 \mathrm{~mm}$ in our experiments). If reconstructions from all SLMs overlap at the same location in space, alignment procedure is satisfactory. Fig. 6 shows the front view of the holographic display system. Continuous array of SLMs can be seen in the figure. Note that the numbers on the SLMs in Fig. 6 are chosen to refer the SLMs in Fig. 5.

In our experiments, we have used computer generated 3D model of a cube (See Fig. 7). It is a wire-frame object. The model consists 


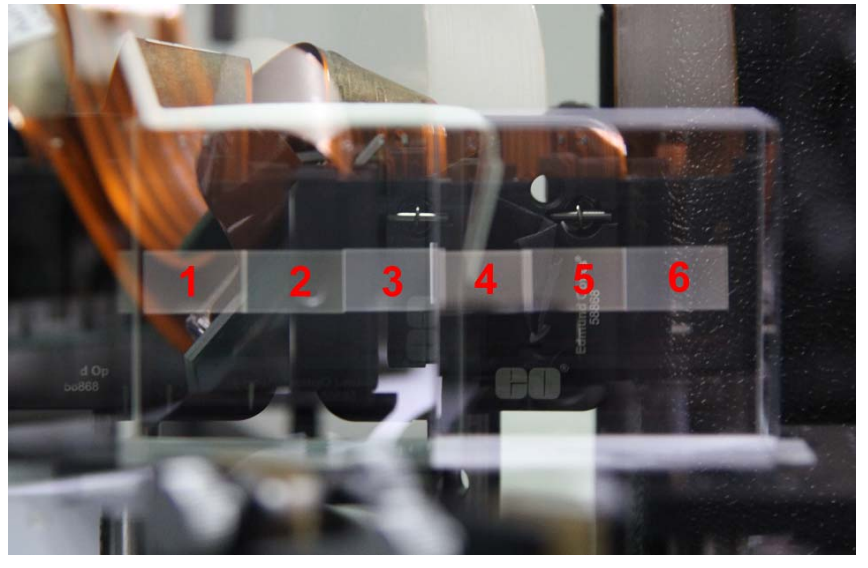

Fig. 6. Front view of the display

of a point cloud. Each point in the point cloud has 3D coordinate and intensity information. Holograms are calculated by using RayleighSommerfeld light propagation algorithm [18]. Each point of the 3D model propagated to the hologram plane. Then, contributions of all the points in the point cloud are superposed to obtain complex field at the hologram plane. Since our SLMs are phase-only, we discard the amplitude and set to unity (plane wave illumination). Although discarding the amplitude lowers the quality, degradations in reconstructions are acceptable. By applying the same procedure, six holograms are calculated one by one, by simply rotating the $3 \mathrm{D}$ object by the corresponding angle. The rotation angles are $-2.5^{\circ},-1.5^{\circ}$, $-0.5^{\circ},+0.5^{\circ},+1.5^{\circ}$ and $+2.5^{\circ}$.

Top view of the setup can be seen in Fig. 8. There are two sets of Holoeye SLM modules [19]. Each SLM module has three phaseonly SLMs with $1920 \times 1080$ pixels each. SLMs in one module are aligned side by side by using non-polarized cubic beam-splitters as described in the previous section (also see Fig. 4. The resultant two sets of wide displays (total of $5760 \times 1080$ pixels each) are also combined by a plate beam-splitter (half-mirror) to obtain a grand total of $11520 \times 1080$ pixels wide holographic display system. All alignments are done with the help of high precision stages.

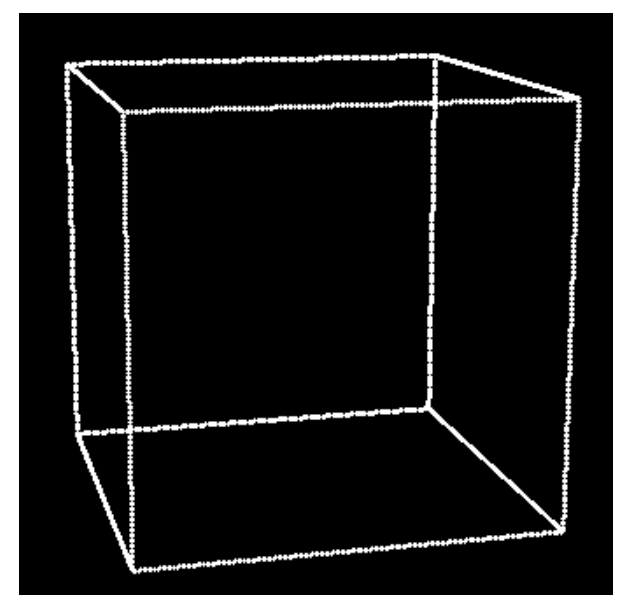

Fig. 7. Computer generated wire-frame 3D model of a cube.

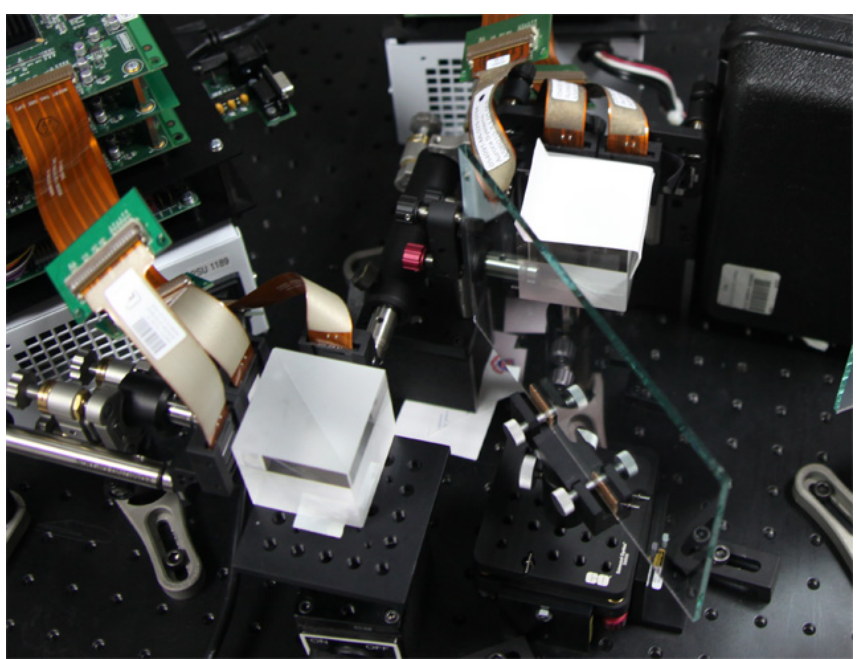

Fig. 8. Top view of the display

\section{EXPERIMENTAL RESULTS}

Fig. 9 shows the optical reconstruction of 3D wire-frame model of a cube. Pictures are taken from different viewing angles (left, center and right). Reconstructions are captured when laser is used as a light source. In order to avoid eye-hazard [20], LED illumination is also used as an alternative source and such reconstructions are observed with naked eye. In addition, since the holographic display is wide in horizontal direction, reconstructions are observed binocularly. Binocular observation increases the 3D perception significantly. Reconstruction distance is approximately $400 \mathrm{~mm}$. We observed that the total field of view increased significantly compared to the single SLM case. The total field of view is approximately $6^{\circ}$. It can be increased up to $18^{\circ}$ if we increase the rotation angle between SLMs to $3^{\circ}$. With the help of the same configuration, we can further increase the field of view and thus viewing angle by using larger number of SLMs and larger beam-splitters.

\section{CONCLUSION}

The proposed system has six phase-only spatial light modulators that are aligned side by side to obtain a $11520 \times 1080$ pixel wide circular holographic display system. All alignments are accomplished by the help of high precision stages. System works both with laser and LED illumination. Optical reconstructions show that increase in the viewing angle is significant compared to the single SLM case. We conclude that circular configuration is superior than the previous planar configurations. We also notice the parallax with the help of the optical reconstructions. Experimental results are satisfactory and

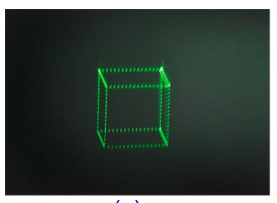

(a)

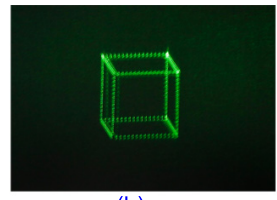

(b)

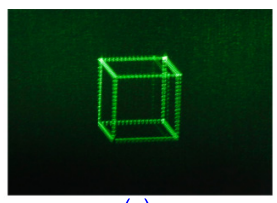

(c)
Fig. 9. Optical reconstruction of 3D wire-frame model of a cube. (a) Left, (b) center, and (c) right views. 
they show that circularly holographic display system works successfully.

\section{ACKNOWLEDGMENTS}

This work is supported by EC within FP7 under Grant 216105 with the acronym Real 3D.

\section{REFERENCES}

[1] M. Kovachev, R. Ilieva, P. Benzie, G. B. Esmer, L. Onural, J. Watson, and T. Reyhan, "Holographic 3DTV displays using spatial light modulators," in Three-Dimensional Television - Capture, Transmission, Display, chapter 15, pp. 529-555. Springer, 2008.

[2] F. Yaraş, H. Kang, and L. Onural, "Real-time phase-only color holographic video display system using led illumination,' Appl. Opt., vol. 48, no. 34, pp. H48-H53, 2009.

[3] F. Yaraş, M. Kovachev, R. Ilieva, M. Agour, and L. Onural, "Holographic reconstructions using phase-only spatial light modulators," in 3DTV Conference: The True Vision - Capture, Transmission and Display of 3D Video, IEEE, 2008.

[4] F. Yaraş and L. Onural, "Color holographic reconstruction using multiple SLMs and LED illumination," in Proc. SPIE., 2009, vol. 7237, p. 72370 O.

[5] F. Yaraş, H. Kang, and L. Onural, "Real-time color holographic video display system," in 3DTV Conference: The True Vision Capture, Transmission and Display of 3D Video, IEEE, 2009.

[6] F. Yaraş, H. Kang, and L. Onural, "Real-time multiple SLM color holographic display using multiple GPU acceleration," in Digital Holography and Three-Dimensional Imaging. 2009, p. DWA4, Optical Society of America.

[7] H. Kang, F. Yaraş, and L. Onural, "Graphics processing unit accelerated computation of digital holograms," Appl. Opt., vol. 48, no. 34, pp. H137-H143, 2009.

[8] H. Kang, F.Yaraş, L. Onural, and H. Yoshikawa, "Real-time fringe pattern generation with high quality," in Digital Holography and Three-Dimensional Imaging. 2009, p. DTuB7, Optical Society of America.

[9] H. Kang, F. Yaraş, and L. Onural, "Quality comparison and acceleration for digital hologram generation method based on segmentation," in 3DTV Conference: The True Vision - Capture, Transmission and Display of 3D Video, IEEE, 2009.

[10] F. Yaraş, H. Kang, and L. Onural, "Multi-SLM holographic display system with planar configuration," in 3DTV Conference: The True Vision - Capture, Transmission and Display of $3 D$ Video, IEEE, 2010.

[11] K. Maeno, N. Fukaya, O. Nishikawa, and T. Honda, "Electroholographic display using 15-megapixel LCD," in Proc. SPIE, Practical Holography X, 1996, vol. 2652, pp. 15-23.

[12] J. Hahn, H. Kim, Y. Lim, G. Park, and B. Lee, "Wide viewing angle dynamic holographic stereogram with a curved array of spatial light modulators," Opt. Express, vol. 16, no. 16, pp. 12372-12386, 2008.

[13] S. Reichelt, R. Haussler, N. Leister, G. Futterer, and A. Schwerdtner, "Large holographic 3D displays for tomorrows TV and monitors - solutions, challenges, and prospects," IEEE Lasers and Electro-Optics Society, 2008. LEOS 2008. 21st Annual Meeting of the, pp. 194-195, 2008.
[14] R. Häussler, A. Schwerdtner, and N. Leister, "Large holographic displays as an alternative to stereoscopic displays," 2008 , vol. 6803 , p. $68030 \mathrm{M}$, SPIE.

[15] S. Reichelt, H. Sahm, N. Leister, and A. Schwerdtner, "Capabilities of diffractive optical elements for real-time holographic displays," 2008, vol. 6912 of Proc. of SPIE, p. 69120P.

[16] A. Schwerdtner, R. Häussler, and N. Leister, "Large holographic displays for real-time applications," 2008, vol. 6912 of SPIE, p. 69120T.

[17] N. Leister, A. Schwerdtner, G. Fütterer, S. Buschbeck, J.-C. Olaya, and S. Flon, "Full-color interactive holographic projection system for large 3D scene reconstruction," 2008, vol. 6911, p. 69110V, SPIE.

[18] J. W. Goodman, Introduction to Fourier Optics, McGraw-Hill Companies, Inc., New York, second edition, 1996.

[19] S. Osten, S. Krger, and A. Steinhoff, "Spatial light modulators based on reflective micro-displays," Technishes Messen, vol. 73, pp. 149-156, 2006.

[20] Y. Barkana and M. Belkin, "Laser eye injuries," Survey of Ophthalmology, vol. 44, no. 6, pp. 459-478, 2000. 